\title{
Earth Station Errors in Two-Way Time and Frequency Transfer
}

\author{
Franklin G. Ascarrunz, S. R. Jefferts, and Thomas E. Parker, Fellow, IEEE
}

\begin{abstract}
We have investigated the earth station errors in two-way time transfer. A $3.7-\mathrm{m}$ earth station and a very small aperture terminal earth station were used in these experiments along with a transponder located on a mountain about $10 \mathrm{~km}$ away. The temperature coefficient of the receive delay for the $3.7 \mathrm{~m}$ NIST earth station was $(-150 \pm 30) \mathrm{ps} / \mathrm{K}$, and the temperature coefficient for the transmit delay was $(-50 \pm 10)$ $\mathrm{ps} / \mathrm{K}$. The overall temperature coefficient for the earth station was $(100 \pm 30) \mathrm{ps} / \mathrm{K}$. Aside from environmental errors there are apparent changes in delay due to characteristics of the modulation/demodulation scheme. Apparent delay changes due to nonlinearity effects in the earth station were as high as $10 \mathrm{~ns}$. The errors due to code dependence and third code interference were sometimes as high as 3 ns.
\end{abstract}

\section{INTRODUCTION}

$\mathbf{T}$ HIS PAPER investigates some of the sources of path nonreciprocity in earth stations. Nonreciprocity in earth station delays remains as one of the largest sources of error in two-way satellite time and frequency transfer (TWSTFT). We have measured the earth station receiver temperature coefficient of delay, as well as the earth station transmitter temperature coefficient of delay. We have also found apparent delay changes due to characteristics of the spread spectrum modulation and demodulation scheme.

\section{Temperature COEFFicient MEASUREMENTS}

A $3.7 \mathrm{~m}$ (NIST) earth station and a very small aperture terminal (VSAT) earth station were used in these experiments along with a transponder located on a mountain about $10 \mathrm{~km}$ away. Two eight-channel data acquisition units were used to record temperature data in real time during two-way sessions. The common clock [1] two-way time transfer sessions were conducted around midnight, when the outside temperature remained fairly constant $( \pm 1 \mathrm{~K})$. Use of a stationary transponder allows the separation of receive (RX) and transmit (TX) components of an earth station since the time delay measured at station A is the sum of the TX delay of station B and the RX delay of station A plus a constant. The temperature dependence of the NIST earth station's RX and TX delays was obtained by raising the temperature of the NIST earth station while the temperature of the VSAT earth station remained nearly constant (Fig. 1). Since the VSAT temperature is constant during the experiment, the RX and TX delays of the VSAT

Manuscript received June 20, 1996; revised October 1, 1996.

The authors are with the National Institute of Standards and Technology, Time and Frequency Division, Boulder, CO 80303 USA.

Publisher Item Identifier S 0018-9456(97)02138-4.

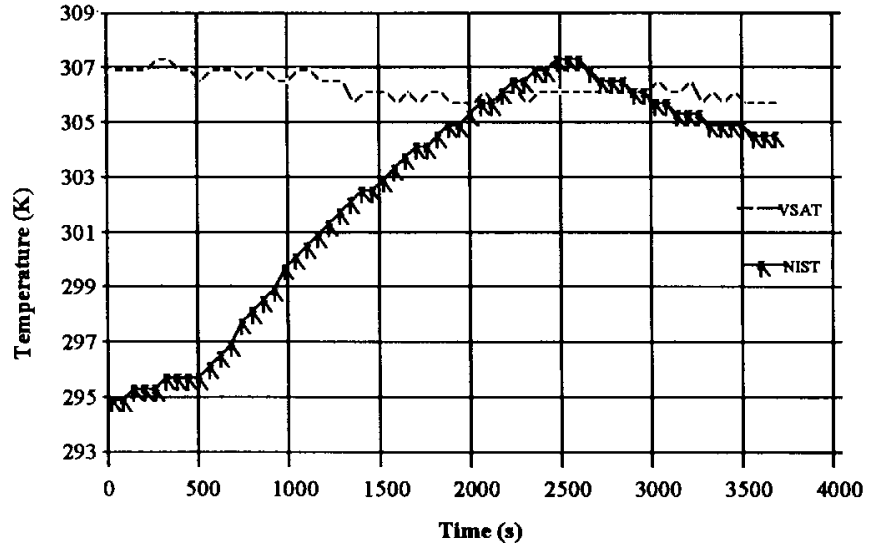

Fig. 1. Temperature of the NIST and VSAT earth stations.

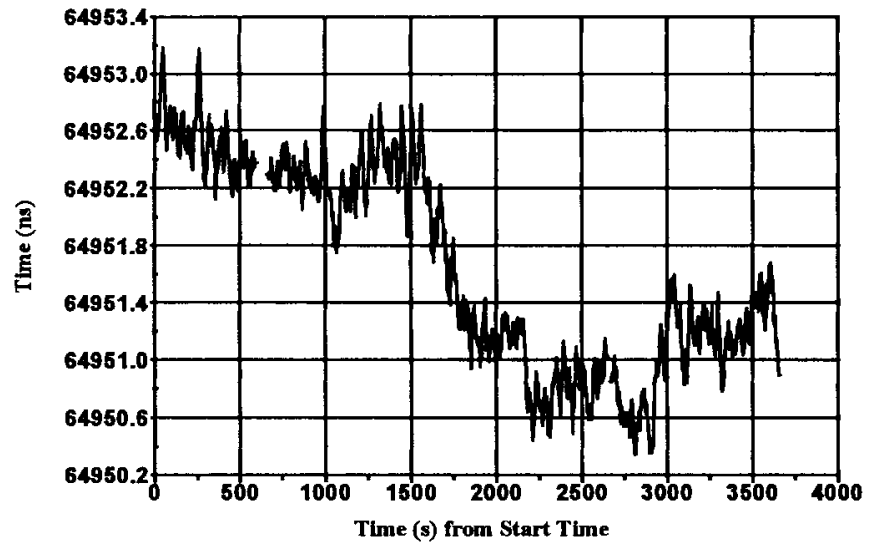

Fig. 2. NIST transmit + VSAT receive delay.

should also remain constant. Figs. 2 and 3 show the temperature dependence of the TX and RX delays of the NIST earth station. The receiver temperature coefficient for the NIST earth station was calculated to be $(-150 \pm 30) \mathrm{ps} / \mathrm{K}$ and the temperature coefficient for the TX delay was $(-50 \pm 10) \mathrm{ps} / \mathrm{K}$. The overall temperature coefficient of the earth station was $(100 \pm 30) \mathrm{ps} / \mathrm{K}$. The overall temperature coefficient of the NIST earth station estimated from a standard common clock two-way satellite time and frequency transfer session using natural temperature variation was $(60 \pm 40) \mathrm{ps} / \mathrm{K}$.

\section{APPARENT ChANGES IN DELAY Due to Signal Distortion}

The up-converter of the NIST earth station was driven into compression during a two-way time transfer session with 


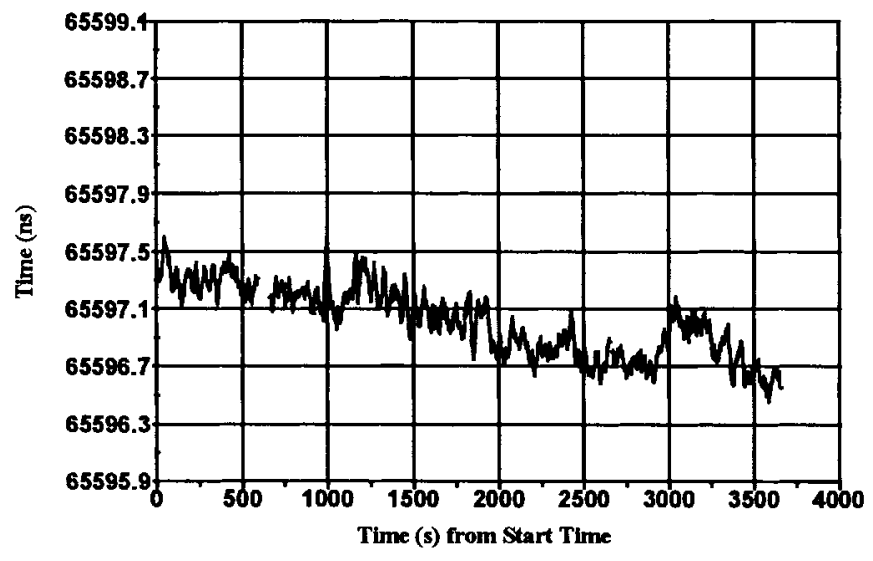

Fig. 3. NIST receive + VSAT transmit delay.

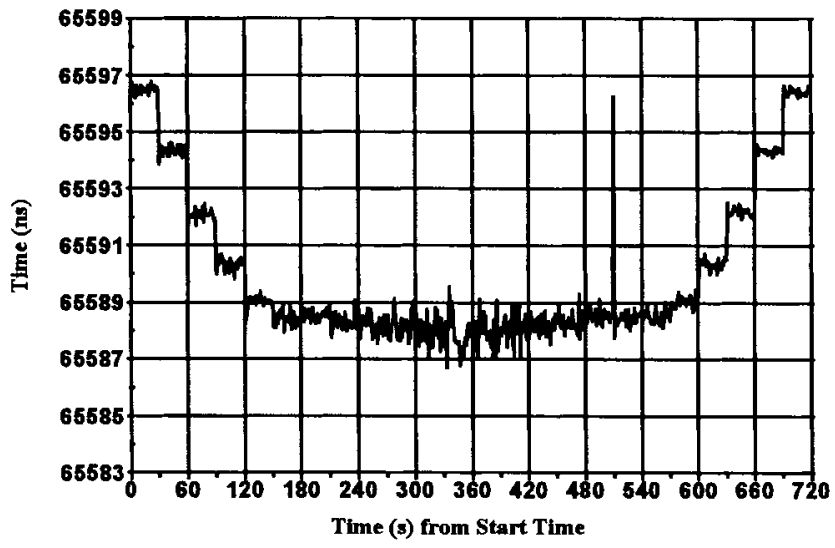

Fig. 4. Apparent changes in NIST TX + VSAT RX delay due to compression of the NIST up-converter. The input to the up-converter is decreased and then increased in $2 \mathrm{~dB}$ steps.

the VSAT earth station via the stationary transponder. The $70 \mathrm{MHz}$ input power to the up-converter was set at $8 \mathrm{~dB}$ above the $1 \mathrm{~dB}$ compression point and decreased in $2 \mathrm{~dB}$ steps until the input level to the up-converter was $12 \mathrm{~dB}$ below the $1 \mathrm{~dB}$ compression point. The power was then increased back up to the value at the beginning of the experiment in $2 \mathrm{~dB}$ steps. The power changes occurred every $30 \mathrm{~s}$ and were implemented with a rotary attenuator with negligible delay change vs. attenuation. The power amplifier following the upconverter remained in linear mode of operation throughout the experiment. The transmit power of the earth station was attenuated to levels similar to those used in conventional TWSTFT. The apparent delay change in the (NIST TX + VSAT RX) delay is shown in Fig. 4. The changes in apparent delay were sometimes as large as $2 \mathrm{~ns} / \mathrm{dB}$ and became very small below the $1 \mathrm{~dB}$ compression point of the upconverter. Similar results were obtained when the upconverter was in a linear mode of operation and the power amplifier in the transmitter was driven into compression. The earth station was operated under gain compression to study the effects of compression on TWSTFT. An earth station used for TWSTFT is not operated under these conditions. It is important to know the effects that gain compression has on TWSTFT because earth station users do not have control over the normal mode

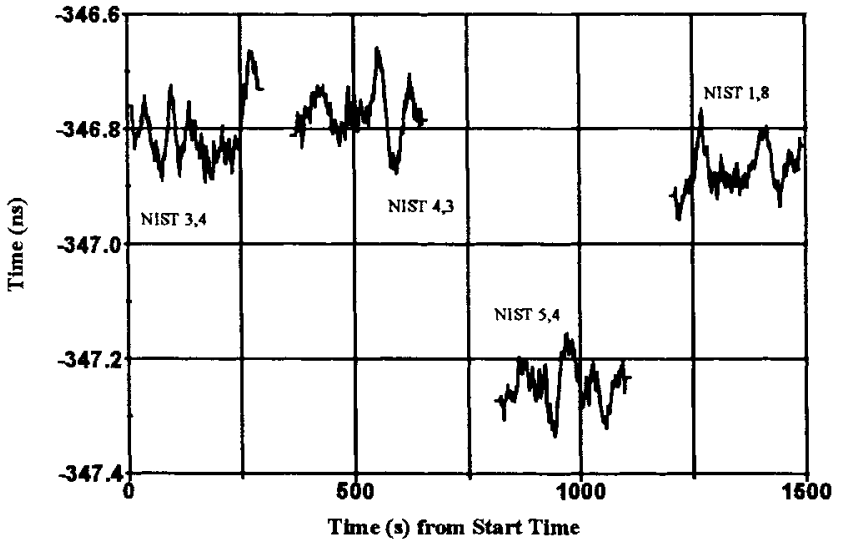

Fig. 5. Apparent delay dependence on spread spectrum code for two-way time and frequency transfer NIST minus VSAT. The NIST codes used were $\mathrm{TX}=3 \mathrm{RX}=4, \mathrm{TX}=4 \mathrm{RX}=3, \mathrm{TX}=5 \mathrm{RX}=4, \mathrm{TX}=1 \mathrm{RX}=8$.

of operation of the satellite transponder. Satellite transponders usually employ traveling wave amplifiers which operate about the $3 \mathrm{~dB}$ compression point. TWSTFT may be sensitive to the satellite transponder user load.

\section{APPARENT Delay DePendence on Spread Spectrum Code}

The transmitter and receiver codes used by the modems ${ }^{1}$ were changed before the beginning of consecutive 5 min twoway time transfer sessions between the NIST and VSAT earth stations using a common clock. For the first run the NIST TX code was set at 3 and the receive code was 4 (NIST 3,4). The other three runs employed the following codes for the NIST earth station were: $\mathrm{TX}=3 \mathrm{RX}=4, \mathrm{TX}=4 \mathrm{RX}=3, \mathrm{TX}=$ $5 \mathrm{RX}=4, \mathrm{TX}=1 \mathrm{RX}=8$. The apparent delay dependence for the sets of codes used is shown in Fig. 5. The apparent delay varied up to $500 \mathrm{ps}$ for the sets of codes used in this experiment.

\section{Apparent Delay Changes Due TO THE PRESENCE OF A THIRD CODE}

Code interference is encountered when more than two earth stations are involved in TWSTFT. These errors are due to coherence among the earth stations participating in TWSTFT [3]. The NIST earth station was configured to do common clock single dish time transfer [1] through the stationary transponder. Modem 1 was set to transmit on code 3 and receive on code 4. Modem 2 was set to transmit on code 4 and receive on code 3 . The VSAT earth station was used to transmit code five to the NIST earth station on $50 \mathrm{~s}$ intervals (off for $50 \mathrm{~s}$, on for $50 \mathrm{~s}$ ). In the first time transfer session the transmitted power from both the VSAT and NIST earth station were equal. During the second session the VSAT

\footnotetext{
${ }^{1}$ The modems used in this investigation were MITREX 2500 modems. The commercial equipment has been identified only to allow other researchers to duplicate results contained here. Such identification does not imply recommendation or endorsement by the National Institute of Standards and Technology. Other commercial equipment may perform differently; in particular, it may be more or less stable than the equipment described here.
} 


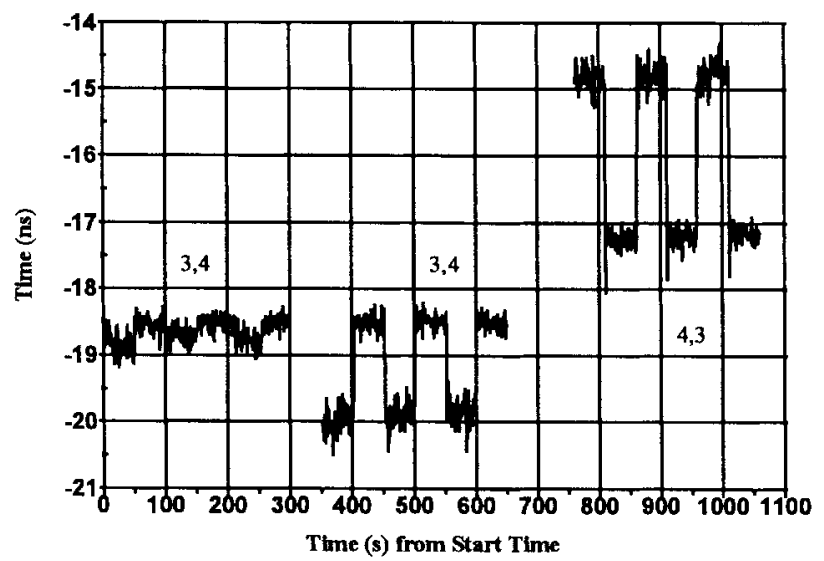

Fig. 6. Apparent delay changes due to the presence of a third interfering code.

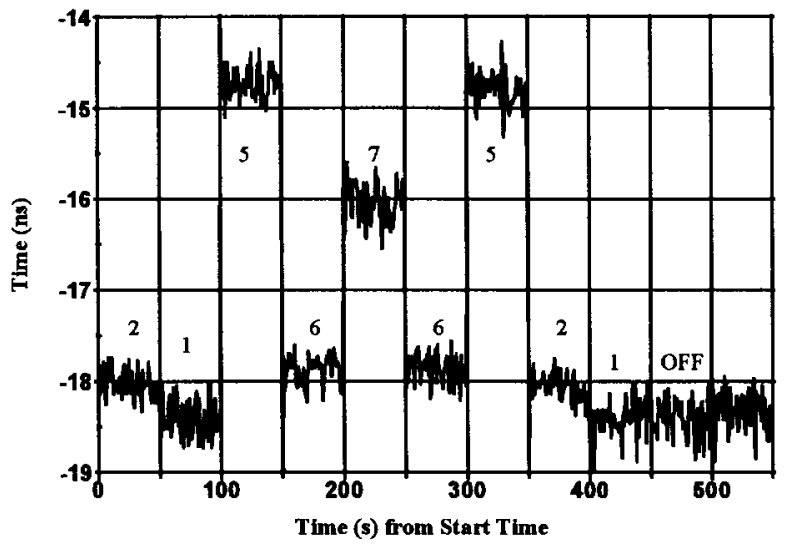

Fig. 7. Apparent delay dependence on the code of a third interfering signal $10 \mathrm{~dB}$ higher than the desired signals.

transmitted power was $10 \mathrm{~dB}$ higher than the NIST transmitted power. During the third session the NIST modems TX and RX codes were reversed but the VSAT transmitted power was still $10 \mathrm{~dB}$ higher. The results of this experiment are displayed in Fig. 6. When the power of the interfering signal was equal in amplitude to that of the desired signal the apparent delay change was about $400 \mathrm{ps}$. In one case the apparent delay change, for an interfering signal $10 \mathrm{~dB}$ higher than the desired signal, was greater than 2 ns.

The apparent delay dependence on the code of an interfering signal is shown in Fig. 7. The NIST earth station was setup to do common-clock, single-dish time transfer through the stationary transponder. The NIST modem TX code was set at 4 and the RX code at 3 . The transmit power of the NIST earth station was about $3 \mathrm{~dB}$ higher than in the previous experiment. The interfering signal was broadcast from the VSAT with the TX code switched from 2 to 7 in 50 s intervals. The VSAT TX power was $10 \mathrm{~dB}$ higher than the NIST TX power.

\section{CONCLUSIONS}

The temperature coefficient of the NIST earth station can lead to large errors in the accuracy of two-way time transfer during the course of a year when temperature fluctuations can be as large as $50 \mathrm{~K}$. Diurnal temperature fluctuations can be as large as $15 \mathrm{~K}$ which would cause fluctuations in delay greater than $1 \mathrm{~ns}$. These errors can be reduced by temperature control of the earth-station electronics or temperature compensation of the two-way data. The errors in delay could also be measured with the use of a satellite simulator and the data adjusted [2]. The problem needs to be addressed by all parties involved in two-way time transfer.

Apparent delay errors as large as $10 \mathrm{~ns}$ can arise from signal distortion from nonlinearity in the earth-station electronics. Nonlinearities could also be present in the satellite transponder. The apparent delay dependence on spread-spectrum code and third-code interference were sometimes as large as $3 \mathrm{~ns}$. This effect would cause TWSTFT to be a function of the number of earth stations participating as well as the codes used and output powers. The errors in delay due to signal distortion, code dependence and third code interference, are not caused by changes in path length through the earth station. These errors are a characteristic of the biphase modulator/demodulator used in the modem. The chip period presently used in the modems is $400 \mathrm{~ns}$ and a $0.1 \%$ change in the correlation function yields a $400 \mathrm{ps}$ error. All of these apparent delay errors should decrease with a higher chip rate. In present systems improvements can be made by minimizing nonlinearities in the earth stations and operating at consistent power. In critical applications it may be necessary to conduct two-way time and frequency transfers with only a pair of earth stations. A consistent and careful approach to two-way frequency transfer is required to minimize stability problems. It will be more difficult to minimize the errors in accuracy for time transfer.

\section{REFERENCES}

[1] C. Hackman, S. R. Jefferts, and T. E. Parker, "Common clock twoway satellite time transfer experiments," in Proc. 1995 IEEE Frequency Control Symp., pp. 275-281.

[2] D. Kirchner, H. Ressler, and R. Robnik, "An automated signal delay monitoring system for a two-way satellite time transfer terminal," in Proc. Europ. Frequency and Time Forum, 1995, pp. 75-79.

[3] D. A. Howe, "Time tracking error in direct-sequence spread spectrum networks due to coherence among signals," IEEE Trans. Commun., vol. 38 , no. $12,1990$.

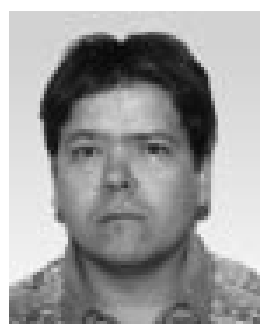

Franklin G. Ascarrunz was born in Rochester, NY, September 21, 1967. He received the B.S and M.S. degrees in aerospace engineering from the University of Colorado, Boulder, in 1989 and 1994, respectively. Through a fellowship with the National Institute of Standards and Technology and the University of Colorado, he is currently pursuing the $\mathrm{PhD}$. degree in aerospace engineering.

Since 1989, he has worked on AM and PM noise measurement systems, AM and PM noise standards, X-Band frequency references for cesium and mercury, and low-noise frequency synthesis. He is currently working on increasing the stability of two-way satellite time and frequency transfer. $\mathrm{He}$ is co-founder of SpectraDynamics, Inc., a firm specializing in ultra-low phase noise synthesis, frequency distribution and metrology in the RF and microwave range. 


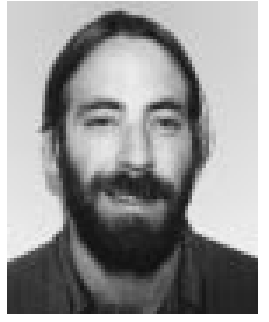

Steven R. Jefferts was born in Seattle, WA, on February 21, 1959. He received the B.S. degree in physics from the University of Washington, Seattle, in 1984, and the Ph.D. degree in atomic physics/precision metrology from the University of Colorado, Boulder, in 1992. He then went to the National Institute of Standards and Technology (NIST), Boulder, CO, as a NRC postdoctoral fellow where he worked on trapped laser cooled ions.

He joined NIST as a permanent staff member in 1994, and presently works on time transfer techniques and primary frequency standards.

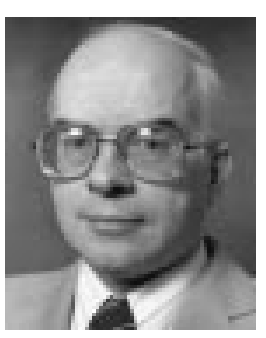

Thomas E. Parker (M'79-SM'86-F'94) was born in Natrona Heights, PA, on September 17, 1945. He received the B.S. degree in physics from Allegheny College, Meadville, PA, in 1967, and the M.S and Ph.D. degrees in physics in 1969 and 1973 respectively, both from Purdue University, West Lafayette, IN.

In August 1973, he joined the Professional Staff, Raytheon Research Division, Lexington, MA. Initially, his work was primarily related to the development of improved temperature stable surface acoustic wave materials. Beginning in 1977, he was responsible for the development of high performance surface acoustic wave (SAW) oscillator technology in the Research Division, including the "All Quartz Package" for SAW devices. His primary interest was frequency stability, with an emphasis on $1 / f$ noise, vibration sensitivity, and long-term frequency stability. In June 1994, he joined the Time and Frequency Divison, National Institute of Standards and Technology (NIST), Boulder, CO. He is the group leader for the Time Scale and Coordination Group and his interests include improved time scales and time transfer technology.

Dr. Parker is a member of Sigma Xi and Sigma Pi Sigma. He has served as an elected member of the Administrative Committee of the IEEE Ultrasonics, Ferroelectrics, and Frequency Control Society (UFFC-S) from 1988 to 1990, and is currently Chair of the Frequency Control Standing Committee of the UFFC-S. He has served on the Technical Program Committees of both the Ultrasonics and the Frequency Control Symposia, and was the Technical Program Chair for the Frequency Control Symposium in 1990 and 1991. He is currently the Associate Editor of Frequency Control-Acoustics of the UFFC$S$ Transactions. He received the 1988 Outstanding Transactions Paper Award from the IEEE Ultrasonics, Ferroelectrics, and Frequency Control Society as a co-author of two papers which appeared in the May and Nov. 1988 issues of IEEE Transactions on Ultrasonics, FerRoelectrics, and FreQuenCy Control. He was the recipient of a Thomas L. Phillips "Excellence in Technology Award" from Raytheon in 1992. In 1994 he received the W. G. Cady Award presented by the IEEE International Frequency Control Symposium. He was the 1996 Distinguished Lecturer of the IEEE Ultrasonics, Ferroelectrics, and Frequency Control Society. 